ISSN 1855-3966 (printed edn.), ISSN 1855-3974 (electronic edn.)

\author{
ARS MATHEMATICA CONTEMPORANEA 18 (2020) 117-126 \\ https://doi.org/10.26493/1855-3974.1852.4f7 \\ (Also available at http://amc-journal.eu)
}

\title{
The distinguishing index of connected graphs without pendant edges*
}

\author{
Wilfried Imrich \\ Montanuniversität Leoben, Franz Josef-Str. 18, 8700 Leoben, Austria \\ Rafał Kalinowski (D), Monika Pilśniak (D), Mariusz Woźniak \\ AGH University of Science and Technology, \\ al. Mickiewicza 30, 30-059 Kraków, Poland
}

Received 13 November 2018, accepted 19 December 2019, published online 24 September 2020

\begin{abstract}
We consider edge colourings, not necessarily proper. The distinguishing index $D^{\prime}(G)$ of a graph $G$ is the least number of colours in an edge colouring that is preserved only by the identity automorphism. It is known that $D^{\prime}(G) \leq \Delta$ for every countable, connected graph $G$ with finite maximum degree $\Delta$ except for three small cycles. We prove that $D^{\prime}(G) \leq$ $\lceil\sqrt{\Delta}\rceil+1$ if additionally $G$ does not have pendant edges.
\end{abstract}

Keywords: Symmetry breaking, distinguishing index of a graph.

Math. Subj. Class. (2020): 05C15, 05E18

\section{Introduction and main result}

We use standard graph theory terminology and notation [3]. In particular, the circumference of a graph $G$, denoted by $c(G)$, is the length of a longest cycle of $G$. A graph that contains a Hamiltonian path, i.e. a path that visits each vertex of the graph, is called traceable. A finite tree is called symmetric (respectively, bisymmetric) if it contains a central vertex $v_{0}$ (resp. a central edge $e_{0}$ ), all leaves are at the same distance from $v_{0}$ (resp. $e_{0}$ ) and all vertices that are not leaves have the same degree. An infinite path $P_{\infty}$ is an infinite connected 2-regular graph.

We consider edge colourings, not necessarily proper. Such a colouring breaks an automorphism $\varphi \in \operatorname{Aut}(G)$ if there exists an edge that is mapped into an edge with a different

\footnotetext{
* The research was partially supported by OEAD grant no. PL 08/2017.

E-mail addresses: imrich@unileoben.ac.at (Wilfried Imrich), kalinows@agh.edu.pl (Rafał Kalinowski), pilsniak@agh.edu.pl (Monika Pilśniak), mwozniak@agh.edu.pl (Mariusz Woźniak)
} 
colour. A colouring is called distinguishing, if it breaks all non-trivial automorphisms. The minimum number of colours in a distinguishing colouring of a graph $G$ is called the distinguishing index of $G$ and is denoted by $D^{\prime}(G)$. Obviously, the distinguishing index is defined only for graphs without $K_{2}$ as a component and with at most one isolated vertex. In this paper, we exclusively consider connected graphs, hence in the sequel, we assume that each graph in question is of order at least 3.

The following general upper bound for the distinguishing index of finite connected graphs was proved in [5].

Theorem 1.1 ([5]). If $G$ is a finite, connected graph of order $n \geq 3$, then

$$
D^{\prime}(G) \leq \Delta(G)
$$

unless $G$ is $C_{3}, C_{4}$ or $C_{5}$.

This result was improved by the third author who characterized all connected graphs with the distinguishing index equal to the maximum degree.

Theorem 1.2 ([9]). Let $G$ be a finite, connected graph of order $n \geq 3$. Then

$$
D^{\prime}(G) \leq \Delta(G)-1
$$

unless $G$ is a cycle, a symmetric or a bisymmetric tree, $K_{4}$ or $K_{3,3}$.

For infinite graphs, a sharp upper bound was proved by Pilśniak and Stawiski in [10].

Theorem 1.3 ([10]). Let $G$ be a connected, infinite graph with finite maximum degree $\Delta(G)$. Then

$$
D^{\prime}(G) \leq \Delta(G)-1
$$

unless $G$ is an infinite path $P_{\infty}$ with $D^{\prime}\left(P_{\infty}\right)=\Delta\left(P_{\infty}\right)=2$.

However, $D^{\prime}(G) \leq 2$ if $G$ belongs to some classes of graphs, e.g. $G$ is a traceable graph of order at least 7 ([9]), $G$ is any Cartesian power of a graph unless $G=K_{2}^{2}$ ([4]), or $G$ is a countable graph every non-identity automorphism of which moves infinitely many vertices ([6]).

Pilśniak [9] formulated the following conjecture.

Conjecture 1.4 ([9]). If $G$ is a 2-connected graph, then

$$
D^{\prime}(G) \leq\lceil\sqrt{\Delta(G)}\rceil+1
$$

We prove this conjecture in a bit stronger form.

Theorem 1.5 (Main result). If $G$ is a connected, countable graph with minimum degree $\delta(G) \geq 2$, then

$$
D^{\prime}(G) \leq\lceil\sqrt{\Delta(G)}\rceil+1 .
$$

In view of Theorem 1.3, the claim obviously holds for countable graphs with infinite $\Delta(G)$. Hence, from now on, we assume that the maximum degree of any graph $G$ in question is finite. 
This result is tight for complete bipartite graphs $K_{2, r^{2}}$ since $D^{\prime}\left(K_{2, r^{2}}\right)=r+1$ for any integer $r \geq 2$. Indeed, there are $r^{2}$ internally disjoint paths of length two between the two vertices $u, v$ of maximum degree in $K_{2, r^{2}}$, and they have to be coloured with distinct ordered pairs of colours. With $r$ colours, we have $r^{2}$ distinct pairs, but if all of them are used, we can transpose $u$ and $v$ and permute the other vertices to obtain an automorphism preserving this colouring. There are also some small graphs $G$ with $D^{\prime}(G)=\lceil\sqrt{\Delta(G)}\rceil+$ 1, e.g. $K_{n}, C_{n}$ for $n \in\{3,4,5\}$, and $K_{3,3}$. We conjecture that the only connected graphs of order at least seven satisfying this equality are complete bipartite graphs $K_{2, r^{2}}$.

The proof of the Main result for 2-connected graphs is given in Section 2 (Theorem 2.2). In Section 3, we prove it for graphs of connectivity 1 (Theorem 3.5).

We believe that the following much stronger claim is true ${ }^{1}$.

Conjecture 1.6. If $G$ is a connected, countable graph with finite minimum degree $\delta \geq 2$, then

$$
D^{\prime}(G) \leq\lceil\sqrt[\delta]{\Delta(G)}\rceil+1
$$

Moreover, for graphs of order at least seven, the equality holds if and only if $G=K_{\delta, r}$, for some positive integer $r$.

If true, this would imply that $D^{\prime}(G) \leq 2$ for every regular graph $G$ of order at least seven. Observe that for vertex-transitive graphs this result would be also implied by the famous Lovász Conjecture [8] that every vertex-transitive graph is traceable since, as it was already mentioned, $D^{\prime}(G) \leq 2$ for every traceable graph of order at least 7 . Let us add that recently Lehner, Pilśniak and Stawiski [7] proved that $D^{\prime}(G) \leq 3$ for every connected, regular graph $G$, finite or infinite.

\section{2-connected graphs}

In the proof of the Main result, we colour the edges of a graph $G$ with colours from the set $Z=\{0,1, \ldots,\lceil\sqrt{\Delta}\rceil\}$. Observe that we always have at least three colours at our disposal. Given a vertex $a$ of a graph $H$, by $\operatorname{Aut}(H)_{a}$ we denote the stabilizer of a vertex $a$, i.e. $\operatorname{Aut}(H)_{a}=\{\varphi \in \operatorname{Aut}(H): \varphi(a)=a\}$. For two vertices $a, b$, we denote $\operatorname{Aut}(H)_{a, b}=$ $\operatorname{Aut}(H)_{a} \cap \operatorname{Aut}(H)_{b}$.

In this section, we prove the Main result for 2-connected, countable graphs. The following lemma plays a key role in the proof.

Lemma 2.1. Let $a, b$ be two vertices of a graph $H$ of finite maximum degree at most $\Delta$, such that

$$
\operatorname{dist}(a, v)+\operatorname{dist}(v, b)=\operatorname{dist}(a, b)
$$

for every vertex $v \in V(H)$. Then $H$ admits an edge colouring with $\lceil\sqrt{\Delta}\rceil$ colours breaking every automorphism of $\operatorname{Aut}(H)_{a, b}$.

Proof. For $r \in\{0,1, \ldots, \operatorname{dist}(a, b)\}$, let

$$
S_{r}(a)=\{v \in V(H): \operatorname{dist}(a, v)=r\}
$$

\footnotetext{
${ }^{1}$ Actually, this conjecture was earlier posed by Pilśniak and Woźniak, and the first part of it was independently formulated by Alikhani and Soltani in [1]. The latter authors claimed a proof therein but we found two errors and a few gaps that we cannot fix.
} 
be the $r$-th sphere centered at the vertex $a$. Thus, for $r<\operatorname{dist}(a, b)$, every vertex $v \in S_{r}(a)$ has at least one and at most $\Delta-1$ neighbours in $S_{r+1}(a)$. For $r \geq 1$, denote $H_{r}=$ $H\left[S_{0}(a) \cup \cdots \cup S_{r}(a)\right]$. We recursively colour the edges between $S_{r}(a)$ and $S_{r+1}(a)$ with $\lceil\sqrt{\Delta}\rceil$ colours such that for each $r$ the following two conditions are satisfied:

(1) $S_{r}(a)$ is fixed pointwise by every automorphism $\varphi \in \operatorname{Aut}(H)_{a, b}$ preserving the colouring of $H_{r+1}$, whenever $S_{r+1}(a)$ is fixed so;

(2) if $A \subseteq S_{r+1}(a)$ is a set of vertices such that there exists a cyclic permutation of $A$ that can be extended to an automorphism $\varphi \in \operatorname{Aut}(H)_{a, b}$ preserving the colouring of $H_{r+1}$, then $|A| \leq\lceil\sqrt{\Delta}\rceil$.

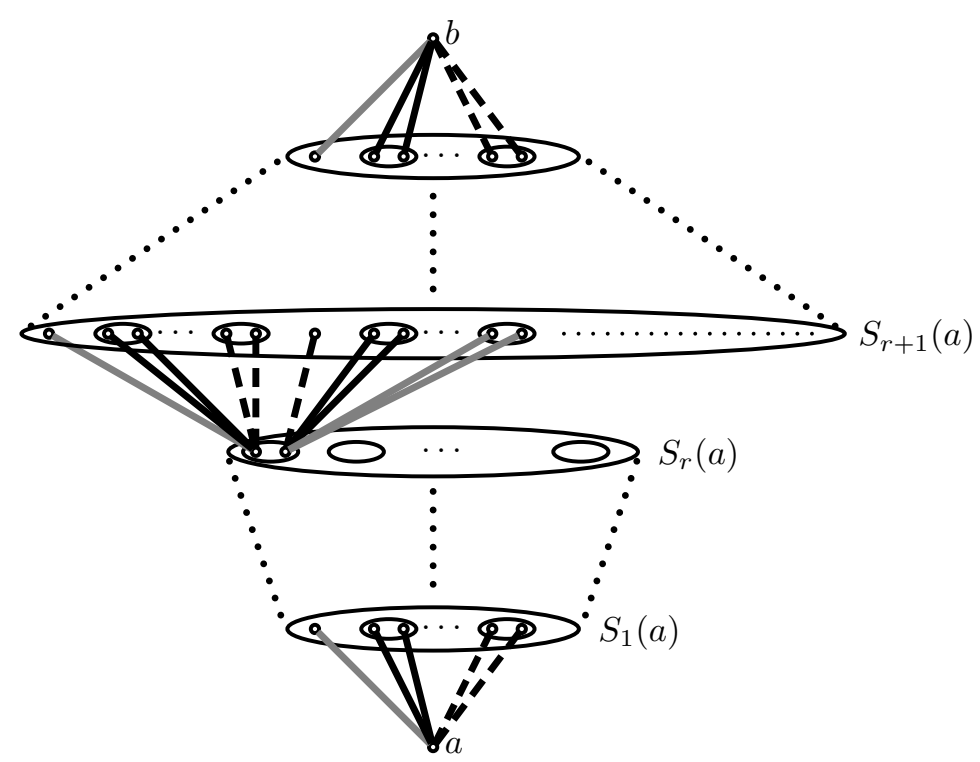

Figure 1: Colouring of edges between subsequent spheres centered at the vertex $a$ breaking all automorphisms of $\operatorname{Aut}_{a, b}(H)$.

First we partition the set of edges incident to $a$ into at most $\lceil\sqrt{\Delta}\rceil$ subsets of cardinality at most $\lceil\sqrt{\Delta}\rceil$, and we colour edges in each subset with the same colour.

Suppose that we have already defined an edge colouring $f$ of $H_{r}$ satisfying the above two conditions for $r-1$ instead of $r$. Let $A=\left\{v_{1}, \ldots, v_{p}\right\}$, with $p \geq 2$, be a set of vertices of $S_{r}(a)$ such that there exists a cyclic permutation of $A$ that can be extended to an automorphism $\varphi \in \operatorname{Aut}(H)_{a, b}$ preserving the colouring $f$. By assumption, $1 \leq|A| \leq$ $\lceil\sqrt{\Delta}\rceil$. Each vertex $v_{i}$ of $A$ has the same number $k \leq \Delta-1$ of incident edges joining it to $S_{r+1}(a)$, and let $a_{j}$ denote the number of those of them that will obtain the colour $j$, for $j \in Z \backslash\{0\}$.

Every vertex $v_{i} \in A$, for $i \in\{1, \ldots, p\}$, can be assigned a distinct sequence $\left(a_{1}, \ldots\right.$, $\left.a_{\lceil\sqrt{\Delta}\rceil}\right)$, where $a_{j} \leq\lceil\sqrt{\Delta}\rceil$ for every $j$. Indeed, take a vertex $v_{i} \in A$ for some $i \in$ $\{1, \ldots, p\}$. If $k \leq\lceil\sqrt{\Delta}\rceil$, then we put $a_{i}=k$ and $a_{j}=0$ for $j \in Z \backslash\{0, i\}$. If $k>(\lceil\sqrt{\Delta}\rceil-1)\lceil\sqrt{\Delta}\rceil$, then we put $a_{i}=k-(\lceil\sqrt{\Delta}\rceil-1)\lceil\sqrt{\Delta}\rceil$, and $a_{j}=\lceil\sqrt{\Delta}\rceil$ for 
$j \in Z \backslash\{0, i\}$. Otherwise, $\lceil\sqrt{\Delta}\rceil+1 \leq k \leq(\lceil\sqrt{\Delta}\rceil-1)\lceil\sqrt{\Delta}\rceil$. If this case, $a_{i}=0$, and for $j \neq i$ we put $a_{j}>0$ such that $\left|a_{j}-\frac{k}{\lceil\sqrt{\Delta}\rceil-1}\right| \leq 1$. Thus, $a_{i} \neq a_{j}$ for $j \neq i$, whence the vertices of $A$ are distinguished.

Moreover, this way we produce at most $\lceil\sqrt{\Delta}\rceil$ vertices in $S_{r+1}(a)$ that can be interchanged by an automorphism preserving the colouring of $H_{r+1}$ because at most $\lceil\sqrt{\Delta}\rceil$ edges joining any vertex of $A$ to $S_{r+1}(a)$ have the same colour.

The second last sphere $S_{r}(a)$, i.e. for $r=\operatorname{dist}(a, b)-1$, has at most $\Delta$ vertices and, due to our construction, any of its subsets $A$ that can be permuted, has at most $\lceil\sqrt{\Delta}\rceil$ vertices. We colour the edges between $b$ and the vertices of $A$ with distinct colours. The unique vertex $b$ of the last sphere is fixed by assumption, hence all spheres are fixed pointwise with respect to any automorphism $\varphi \in \operatorname{Aut}(H)_{a, b}$ preserving the edge colouring $f$ of $H$.

Finally, we colour edges within each sphere with an arbitrary colour.

Given a cycle $C$ of a 2-connected graph $G \neq C_{4}$ and two distinct colours $\alpha, \beta \in$ $Z \backslash\{0\}$, by $C_{0}(\alpha, \beta)$ we denote this cycle coloured such that three consecutive edges are coloured with $\alpha, 0, \beta$ in that order, and all other edges of the cycle are coloured with 0 . Exceptionally, in case $G=C_{4}$, by a colouring $C_{0}(\alpha, \beta)$ of $C_{4}$ we mean the colouring $\alpha, \beta, 0,0$ of its consecutive edges.

Theorem 2.2. If $G$ is a 2-connected, countable graph with finite maximum degree $\Delta$, then

$$
D^{\prime}(G) \leq\lceil\sqrt{\Delta}\rceil+1
$$

Proof. If the circumference of $G$ equals 3, then $G=C_{3}$ and the claim holds.

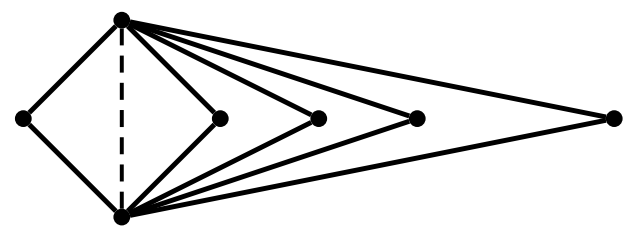

Figure 2: For a given $n \geq 5$, there are exactly two non-isomorphic graphs of order $n$ and circumference 4 (the dashed edge may exist or not).

Let $c(G)=4$. If $G \in\left\{C_{4}, K_{4}, K_{4}-e\right\}$, then $D^{\prime}(G) \leq 3$, and the claim is true. Suppose $|V(G)| \geq 5$. Then either $G=K_{2, \Delta}$ or $G=K_{2, \Delta-1}+e$, where $e$ is an edge between the two vertices of maximum degree $\Delta \geq 3$ (see Figure 2). Indeed, let $V(G)=$ $\left\{u_{1}, \ldots, u_{n}\right\}$ with $n \geq 5$, and suppose that $u_{1}, u_{2}, u_{3}, u_{4}$ are consecutive vertices of a cycle $C$ of length 4 . As $G$ is 2 -connected and $c(G)=4$, all other vertices $u_{5}, \ldots, u_{n}$ have to be adjacent to the same pair of non-consecutive vertices of $C$, say $u_{1}, u_{3}$. Thus, we get a complete bipartite graph $K_{2, n-2}$. The only possible additional edge not violating the condition $c(G)=4$, is $e=u_{1} u_{3}$. As it was mentioned in the Introduction, in such a graph $G \in\left\{K_{2, \Delta}, K_{2, \Delta-1}+e\right\}$, we have enough colours for a distinguishing colouring of $G$. It is also easy to see that we can require that colour 0 appears only in one cycle of length four, coloured as $C_{0}(\alpha, \beta)$.

Suppose then that $G$ has a cycle $C$ of length at least 5 . We colour it as $C_{0}(\alpha, \beta)$ for some distinct $\alpha, \beta \in Z \backslash\{0\}$. Moreover, we colour all chords of $C$ with $\alpha$. We can require 
that the colour 0 appears only in this cycle $C$. Thus each vertex of $C$ is fixed by every automorphism of $G$ preserving the colouring. To see this it is enough to observe that both endpoints of the unique edge coloured with $\beta$ are fixed.

We define a colouring of the remaining edges of $G$ recursively, as follows. Suppose that we have already coloured the edges of an induced 2-connected subgraph $F$ of $G$ such that the only automorphism of $G$ preserving this colouring is the identity, regardless of a colouring of the edges outside $F$. Thus $F=G[V(C)]$ in the initial step. If $F \neq G$, then we choose a shortest path $P$ between any two vertices of $F$ containing at least one vertex outside $F$. Let $a, b$ be the end-vertices of $P$, and let $p$ be the length of $P$. As $P$ is shortest possible, each path of length $p$ between $a$ and $b$ in $G$ is either uncoloured or fully coloured. Consider the subgraph $H$ induced by the vertices of $P$ and of all uncoloured paths between $a$ and $b$ of length $p$. Thus, all edges of $H$ are uncoloured yet. We colour the edges of $H$ with $\lceil\sqrt{\Delta}\rceil$ colours according to Lemma 2.1. Let $F^{\prime}=G[V(F) \cup V(H)]$. We thus obtained a colouring of all edges of $F^{\prime}$ that breaks every non-trivial automorphism of $G$, because the colouring of $F$ already break them.

If $F^{\prime} \neq G$, the we repeat this procedure with $F^{\prime}$ instead of $F$ until all edges of $G$ are coloured.

Actually, we have proved the following result which we use in the next section.

Corollary 2.3. Let $G$ be a 2-connected, countable graph with finite maximum degree $\Delta$ and let $C$ be a longest cycle or any cycle of length at least five in $G$. Then for every two distinct colours $\alpha, \beta \in Z \backslash\{0\}$, any colouring $C_{0}(\alpha, \beta)$ of its edges can be extended to a distinguishing colouring of $G$ with colours from the set $Z$ such that all edges coloured with 0 belong to the cycle $C$.

Proof. The claim follows directly from the proof of Theorem 2.2 unless $|G|=4$. In the latter case, $Z=\{0,1,2\}$, and $G$ contains a cycle $C_{4}$, which we colour as $C_{0}(1,2)$ (recall that this means a colouring $\alpha, \beta, 0,0$ of consecutive edges if $G=C_{4}$, and $\alpha, 0, \beta, 0$ otherwise). This is clearly a distinguishing colouring of $G=C_{4}$. If $G=K_{4}$, then the other two edges get distinct colours 1,2. Otherwise, $G=K_{4}-e$, and the chord of $C_{4}$ gets colour 1 .

\section{Graphs of connectivity 1}

Observe that it follows from Theorem 1.2 and Theorem 1.3 that Theorem 1.5 is true for graphs of maximum degree $\Delta \leq 5$ satisfying the conditions of the Theorem. This fact did not matter in the proof for 2-connected graphs, but it facilitates a bit our proof for graphs with cut vertices. From now on, assume that $G$ is a countable graph of finite maximum degree $\Delta \geq 6$ and connectivity 1 . Hence, we have at least four colours $0,1,2,3$ at our disposal.

Two edge colourings $f_{1}, f_{2}$ of a graph $H$ are called isomorphic with respect to a group $\Gamma \subseteq \operatorname{Aut}(H)$ if there exists an automorphism $\varphi \in \Gamma$ such that $f_{1}(u v)=f_{2}(\varphi(u) \varphi(v))$ for every edge $u v \in E(H)$. Furthermore, $f_{1}, f_{2}$ are called isomorphic if they are isomorphic with respect to $\operatorname{Aut}(H)$.

Lemma 3.1. Let $u_{0}$ be a vertex of a 2-connected, countable block $H_{0}$ of finite maximum degree at most $\Delta$, where $\Delta \geq 6$. Then $H_{0}$ admits at least $\lceil\sqrt{\Delta}\rceil$ edge colourings with colours from the set $Z$, breaking all non-trivial automorphisms of $\operatorname{Aut}\left(H_{0}\right)_{u_{0}}$, which are pairwise non-isomorphic with respect to $\operatorname{Aut}\left(H_{0}\right)_{u_{0}}$, and do not contain $C_{0}(1,2)$. 
Proof. First observe that if $H_{0}$ is a 2-connected, countable graph and $c\left(H_{0}\right) \geq 5$, then every vertex of $H_{0}$ belongs to a cycle of length at least five. This easily follows from the fact that every vertex outside a given cycle $C$ of length at least five is the origin of two internally disjoint paths to two vertices of $C$, which partition the cycle $C$ into two paths, one of which is of length at least three.

We choose a longest cycle $C$ in $B$ passing through the vertex $u_{0}$ if $B$ is finite, or a cycle of length at least five if $B$ is infinite. It is easy to see that $C$ is a longest cycle in $G$ whenever $|C| \leq 4$. We colour $C$ as $C_{0}(\alpha, \beta)$, for $\{\alpha, \beta\} \neq\{1,2\}$. By Corollary 2.3, regardless of location of colours $\alpha$ and $\beta$ on $C$, this colouring can be extended to a distinguishing colouring of $H_{0}$ using colours from $Z \backslash\{0\}$. We have $\left(\begin{array}{c}\lceil\sqrt{\Delta}\rceil \\ 2\end{array}\right)-1$ choices for the set of two colours $\{\alpha, \beta\} \neq\{1,2\}$. For a given colouring $C_{0}(\alpha, \beta)$, there are at least three possible placements for $u_{0}$ (actually, there are more possibilities if $\left|H_{0}\right|>3$ ). Hence, there are at least $L=3\left(\left(\begin{array}{c}\lceil\sqrt{\Delta}\rceil \\ 2\end{array}\right)-1\right)$ edge colourings of $H_{0}$ that are pairwise non-isomorphic with respect to $\operatorname{Aut}\left(H_{0}\right)_{u_{0}}$. The inequality $L \geq\lceil\sqrt{\Delta}\rceil$, equivalent to $3(\lceil\sqrt{\Delta}\rceil)^{2}-5\lceil\sqrt{\Delta}\rceil-6 \geq$ 0 , holds whenever $\lceil\sqrt{\Delta}\rceil) \geq 3$.

Lemma 3.2. Let $H_{0}$ be a graph of finite maximum degree at most $\Delta$, with $\Delta \geq 6$, consisting of $s \geq 2$ copies of a 2 -connected block $B$ sharing a common cut vertex $u_{0}$. Then $H_{0}$ admits at least $\lceil\sqrt{\Delta}\rceil$ pairwise non-isomorphic distinguishing edge colourings with colours from the set $Z$, such that $C_{0}(1,2)$ does not appear in any of these colourings.

Proof. Let $H_{0}$ be a graph satisfying the assumptions. Observe that $\operatorname{Aut}\left(H_{0}\right)=\operatorname{Aut}\left(H_{0}\right)_{u_{0}}$ for $s \geq 2$. Clearly, $s \leq\left\lfloor\frac{\Delta}{2}\right\rfloor$.

As we have shown above, the block $B$ admits at least $L=3\left(\left(\begin{array}{c}\lceil\sqrt{\Delta}\rceil \\ 2\end{array}\right)-1\right)$ colourings which are pairwise non-isomorphic with respect to $\operatorname{Aut}\left(H_{0}\right)_{u_{0}}$. To colour the graph $H_{0}$, we select $s$ of them. Hence, we have at least $\left(\begin{array}{l}L \\ s\end{array}\right)$ pairwise non-isomorphic colourings of $H_{0}$. Clearly, $\left(\begin{array}{l}L \\ s\end{array}\right) \geq L$, when $1 \leq s \leq\left\lfloor\frac{\Delta}{2}\right\rfloor \leq L-1$, which is the case for $\Delta \geq 6$. We know that $L \geq\lceil\sqrt{\Delta}\rceil$, hence there are at least $\lceil\sqrt{\Delta}\rceil$ non-isomorphic distinguishing colourings of $H_{0}$.

Lemma 3.3. Let $H_{0}$ be a graph satisfying the assumptions of Lemma 3.1 or of Lemma 3.2. Let $T$ be a symmetric tree of order at least 3 with a central vertex $v_{0}$. A graph $H$ is obtained by attaching a copy of the graph $H_{0}$ to every leaf of $T$ in such a way that each pendant edge of $T$ is incident to the same vertex $u_{0}$ of $H_{0}$. If the maximum degree of $H$ is at most $\Delta$ and $\Delta \geq 6$, then there exists a distinguishing colouring of $H$ with colours from the set $Z$, and without $C_{0}(1,2)$.

Proof. We colour the edges of $T$ with $\lceil\sqrt{\Delta}\rceil$ colours similarly as in the proof of Lemma 2.1. That is, at most $\lceil\sqrt{\Delta}\rceil$ edges incident to the central vertex $v_{0}$ of $T$ get the same colour. Then recursively, at each level of $T$, any set $A$ of vertices that can be cyclically permuted by an automorphism of $H$ preserving the hitherto defined colouring, has at most $\lceil\sqrt{\Delta}\rceil$ elements. For each vertex $v$ of $A$, we choose a distinct colour $i \in Z$ such that the number of edges joining $v$ to the next level of $T$ and coloured with $i$, is different from the number of such edges coloured with any other colour. We arrive at the leaves of $T$ with a colouring such that every set $A$ of leaves that can be cyclically permuted by an automorphism of $T$ preserving the colouring of $T$ has at most $[\sqrt{\Delta}\rceil$ elements. It follows from Lemma 3.1 or Lemma 3.2 (depending on the structure of $H_{0}$ ) that each copy of $H_{0}$ attached at a leaf 




Figure 3: Example of a 4-colouring of a graph $H$ of Lemma 3.3 with $\Delta=9$, where a graph $H_{0}$ consisting of four triangles sharing a cut vertex $u_{0}$ is attached to every leaf of a tree $T=K_{1,9}$.

belonging to the same set $A$ has a distinct colouring satisfying our assumptions. Thus we obtain a desired colouring of the whole graph $H$.

The following result of Broere and Pilśniak [2] will be useful in the next proof.

Theorem 3.4 ([2]). If $T$ is an infinite tree without leaves, then $D^{\prime}(T) \leq 2$.

We are now ready to prove Theorem 1.5 for graphs with cut vertices.

Theorem 3.5. Let $G$ be a countable graph of connectivity 1 and without pendant edges. If $G$ has finite maximum degree $\Delta$, then

$$
D^{\prime}(G) \leq\lceil\sqrt{\Delta}\rceil+1
$$

Proof. Recall that we may assume that $\Delta \geq 6$, whence the set $Z$ contains at least four colours. If $G$ is a tree, then it has no leaves, so $D^{\prime}(G) \leq 2$ by Theorem 3.4.

Suppose then that $G$ contains a 2 -connected block $B_{0}$. Let $C$ be a longest cycle of $B_{0}$, if it exists, or any cycle of length at least five of $B_{0}$. Colour it with $C_{0}(1,2)$. By Corollary 2.3, there is a distinguishing edge colouring of $B_{0}$, where colour 0 is used only on the cycle $C$. In our colouring of the whole graph $G$, we ensure that $C_{0}(1,2)$ does not appear again, therefore each vertex of $B_{0}$ is fixed by every automorphism of $G$ preserving the colouring of $B_{0}$. We recursively extend this colouring in such a way that every vertex with at least one coloured incident edge is fixed as well.

Now, we set any ordering $v_{1}, v_{2}, \ldots$ of all cut vertices of $G$. We recursively consider the first vertex $v_{i}$ in this ordering that belongs to an already coloured block, as well as to an uncoloured one. Hence, $v_{i}$ is already fixed by every $\varphi \in \operatorname{Aut}(G)$ preserving the existing partial colouring. We proceed with $v_{i}$ in four stages. 
1. Let $B_{1}, \ldots, B_{l}$ be pairwise non-isomorphic, uncoloured 2-connected blocks containing $v_{i}$. For each $j \in\{1, \ldots, l\}$, we consider a maximal subgraph $H_{0}$ of $G$ that consists of $s \geq 1$ copies of $B_{j}$ sharing the cut vertex $v_{i}$. Taking $u_{0}=v_{i}$, by Lemma 3.1 if $s=1$, or by Lemma 3.2 if $s \geq 2$, there is a distinguishing colouring of $H_{0}$ with colours from the set $Z$, not containing $C_{0}(1,2)$.

2. We consider every uncoloured maximal subgraph $H$ satisfying the assumptions of Lemma 3.3. We colour $H$ distinguishingly according to the conclusion of Lemma 3.3. Consequently, all 2-connected blocks containing $v_{i}$ are now coloured.

3. If some components of $G-v_{i}$ are uncoloured infinite trees, then we consider the infinite tree $T$ containing all those components and the vertex $v_{i}$, and we colour the edges of $T$ with two colours, by Theorem 3.4.

4. We colour every yet uncoloured edge $e$ incident to $v_{i}$ arbitrarily. Note that both endpoints of $e$ are already fixed by any $\varphi \in \operatorname{Aut}(G)$ preserving the existing partial colouring, because uncoloured components of $G-v_{i}$ are pairwise non-isomorphic.

This recursive procedure yields a distinguishing colouring of $G$ with $\lceil\sqrt{\Delta}\rceil+1$ colours, which completes the proof of Theorem 3.5, and thus of Theorem 1.5.

\section{ORCID iDs}

Wilfried Imrich (D) https://orcid.org/0000-0002-0475-9335

Rafał Kalinowski (D) https://orcid.org/0000-0002-3021-7433

Monika Pilśniak (D) https://orcid.org/0000-0002-3734-7230

Mariusz Woźniak (D) https://orcid.org/0000-0003-4769-0056

\section{References}

[1] S. Alikhani and S. Soltani, Relationship between the distinguishing index, minimum degree and maximum degree of graphs, arXiv:1705.05758 [math.CO].

[2] I. Broere and M. Pilśniak, The distinguishing index of infinite graphs, Electron. J. Combin. 22 (2015), \#P1.78 (10 pages), doi:10.37236/3933.

[3] R. Diestel, Graph Theory, volume 173 of Graduate Texts in Mathematics, Springer, Berlin, 5th edition, 2018.

[4] A. Gorzkowska, R. Kalinowski and M. Pilśniak, The distinguishing index of the Cartesian product of finite graphs, Ars Math. Contemp. 12 (2017), 77-87, doi:10.26493/1855-3974.909. $0 \mathrm{e} 1$.

[5] R. Kalinowski and M. Pilśniak, Distinguishing graphs by edge-colourings, European J. Combin. 45 (2015), 124-131, doi:10.1016/j.ejc.2014.11.003.

[6] F. Lehner, Breaking graph symmetries by edge colourings, J. Comb. Theory Ser. B 127 (2017), 205-214, doi:10.1016/j.jctb.2017.06.001.

[7] F. Lehner, M. Pilśniak and M. Stawiski, A bound for the distinguishing index of regular graphs, European J. Combin. 89 (2020), 103145 (9 pages), doi:10.1016/j.ejc.2020.103145.

[8] L. Lovász, Problem 11, in: Combinatorial Structures and Their Applications, Gordon and Breach, New York-London-Paris, 1970, proceedings of the Calgary International Conference on Combinatorial Structures and their Applications held at the University of Calgary, Calgary, Alberta, Canada, June. 
[9] M. Pilśniak, Improving upper bounds for the distinguishing index, Ars Math. Contemp. 13 (2017), 259-274, doi:10.26493/1855-3974.981.ff0.

[10] M. Pilśniak and M. Stawiski, The optimal general upper bound for the distinguishing index of infinite graphs, J. Graph Theory 93 (2020), 463-469, doi:10.1002/jgt.22496. 